\title{
Tumor cell-specific Serpin A1 expression in vulvar squamous cell carcinoma
}

Lagerstedt $\mathbf{M}^{\mathrm{a}, \mathrm{b}}$, Huotari-Orava $\mathbf{R}^{\mathrm{b}, \mathrm{c}}$, Nyberg $\mathbf{R}^{\mathrm{d}}$, Nissinen Le,f, Farshchian $\mathbf{M}^{\mathrm{e}, \mathrm{f}}$, Laasanen S-La, Snellman E $E^{\mathrm{a}, \mathrm{b}}$, Mäenpää JU ${ }^{\mathrm{b}, \mathrm{d}}$, Kähäri V-Me,

a. Department of Dermatology, Tampere University Hospital, Finland, b. Faculty of Medicine and Life Sciences, University of Tampere, Finland, c. Fimlab Laboratorie

\section{OBJECTIVES}

Two main inflammatory etiologies precede vulvar squamous cell carcinoma (vSCC), chronic autoimmune dermatitis lichen sclerosus (LS) or high risk human papillomavirus (HPV) (1). Serpin A1, also called $\alpha 1$-antitrypsin, is a serine protease inhibitor playing a role in immunology and tumorigenesis (2). Serpin A1 is also a marker of poor prognosis is various cancer types (3). The tumor microenviroment of $v S C C$ is largely unknown .

The aim of this study was to evaluate the role of Serpin A1 in lichen sclerosus and vSCC by $\mathrm{ICH}$ and serum analysis.

\section{METHODS}

ICH study of Serpin A1 included 120 samples from 76 patients: 18 normal vulvar skin, 53 LS, 9 carcinoma in situ (dVIN/HSIL) and 40 vSCC samples (24 LSdependent and 16 LS-independent/HPV-dependent vSCC).

Analysis of Serpin A1 serum concentrations included serum samples from 29 vSCC patients before surgical treatment, 15 vSCC patients in follow-up after surgical treatment (av. 2,6 years), 30 LS, and 10 age-matched control patients. These patients had no history of other tumors, acute infections or other inflammatory diseases.

\section{RESULTS}

Tumor cell-specific Serpin A1 overexpression was detected in $88 \%$ of vSCC samples independent on presence of LS. Intensity of Serpin A1 expression was significantly higher in vSCC than in healthy vulvar skin , LS or dVIN/HSIL (Figure 1A-E). LS without malignant progression was more often Serpin A1 positive than LS without malignant progression. Intensity of Serpin A1 did not correlate to overall survival of vSCC patients. Serum concentrations of Serpin A1 did not differ between vSCC, LS or control groups.

Figure 1. Serpin A1 expressions in

A) healthy vulvar skin,

B) lichen sclerosus,

C) differentiated vulvar intraepithelial neoplasia (dVIN), and D)vulvar squamous cell carcinoma (vSCC). E)

Semiquantative analysis on Serpin A1 expression, ${ }^{* * *} p<0.001$, ${ }^{* *} p<0.01,{ }^{*} p<0,05$ ( $x^{2}$ test).
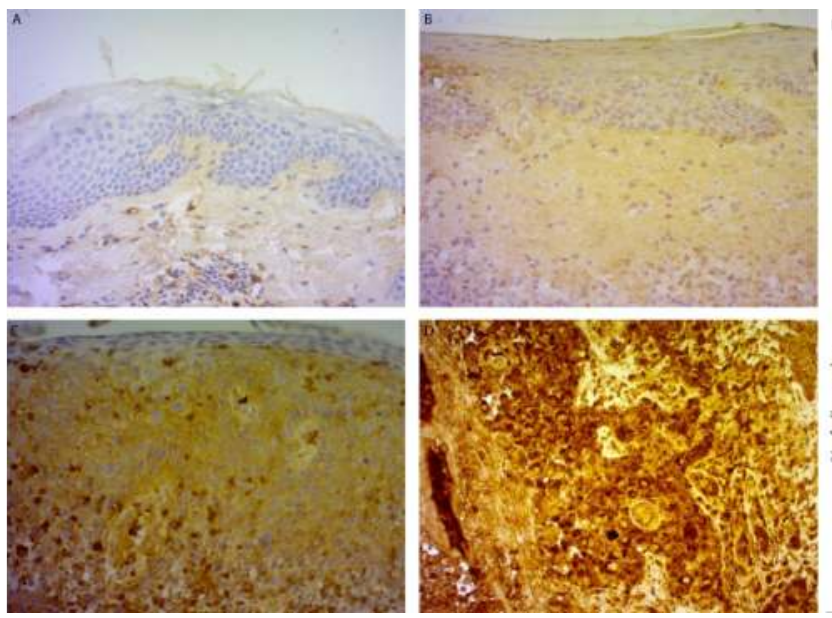

\section{CONCLUSIONS}

Tumor cell-specific Serpin A1 overexpression is associated with vSCC independent of the etiology of vSCC, which confirms the inflammation-induced nature of vSCC. Serpin A1 seems to lack prognostic value in LS and VSCC patients. Our study brought a novel marker , Serpin A1, to microenviroment of vSCC.

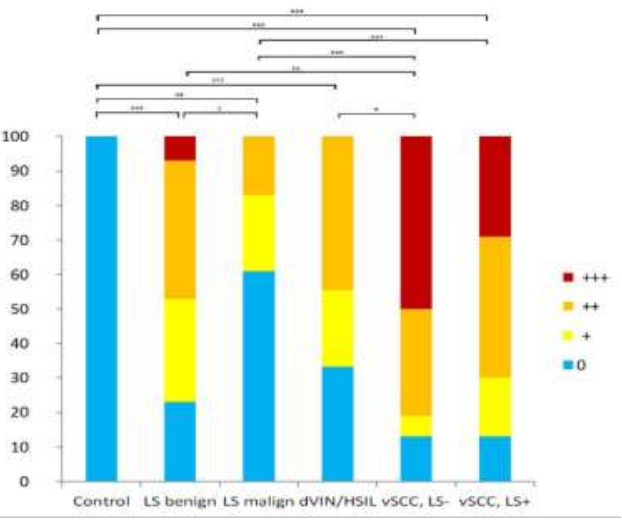

\section{REFERENCES}

1. Del Pino M, Rodriguez-Carunchio L, Ordi J. Pathways of vulvar intraepithelial neoplacia and squamous cell carcinoma. Histopathology 2013; 62: 161-175.

2. Ehlers MR. Immuno-modulating effects of alpha-1 antitrypsin. Biol Chem 2014; 10: 1187-1193.

3. Farshchian M, Kivisaari A, Ala-aho R, et al. Serpin peptidase inhibitor clade A member 1 (Serpin A1) is a novel biomarker for progression of cutaneous squamous cell carcinoma. Am J Pathol 2011; 179 : 1110-1119. 